\title{
Making Healthcare Systems More Efficient and Sustainable in Emerging and Developing Economies Through Disruptive Innovation: The Case of Nigeria
}

\author{
Ivo Pezzuto ${ }^{1}$ \\ ${ }^{1}$ International School of Management, Paris, France \\ Correspondence: Ivo Pezzuto, International School of Management, Paris, France. E-mail: \\ ivo.pezzuto@faculty.ism.edu
}

Received: May 18, 2019 Accepted: June 10, 2019 Online Published: August 29, 2019

doi:10.5539/jms.v9n2p1 URL: https://doi.org/10.5539/jms.v9n2p1

\begin{abstract}
This paper focuses on the potential opportunities that disruptive innovation may bring to the healthcare sector of emerging and developing economies, and in particular to the one of the leading Sub-Saharan Africa's country, Nigeria. The author examines the possibility of using advancements in the innovation of Technology 4.0 to bridge the gap in access to what could be defined as "good enough" healthcare services for poorer regions of the world while also aiming to potentially reduce healthcare costs and making the local healthcare systems more sustainable, productive, and accessible. Nigerian health industry is used as an exploratory case study to examine the feasibility of implementing Mobile Health and Telehealth Systems, and more in general, to assess the potential benefits of disruptive innovations in the healthcare industry for the lower income patients of emerging and developing economies. This analysis on disruptive innovation, industry competitiveness, and sustainability of the healthcare models is inspired by Michael Porter's Creating Shared Value (CSV) strategic framework (Porter et al., 2011; 2018) and by Clayton Christensen's Disruptive Innovation Theory (Christensen et al., 1997; 2000; 2004; 2006; 2013; 2015, 2017). This study also aims to provide a compelling argument supporting the thesis that disruptive innovations in the healthcare system can help grant access to critical basic healthcare services in poor regions of the world while also achieving multiple goals such as, sustainability, efficiency, shared-value creation, and corporate profitability for forward-looking firms with scalable and disruptive business models. Ultimately, the paper aims to contribute to the body of knowledge in the field of disruptive innovation, sustainability, and creating shared-value strategies, assessing the feasibility of solutions that may drive to improved competitiveness, social progress, social inclusion, and sustainability of the healthcare industry in one of the developing economies. The results of this study aim to prove that, in the coming years, disruptive innovations are likely to redefine the competitive environment of the healthcare industry and improve the healthcare conditions of the poorer, underserved, and underreached population of developing and emerging economies like Nigeria, thus increasing their life expectancy rates.
\end{abstract}

Keywords: disruptive innovation, remote patient monitoring, home health, health industry, telemedicine, digital medicine, digital innovation, mobile health, creating shared value strategies

\section{Introduction}

\subsection{The Importance of the Problem}

The Nigerian healthcare sector is currently experiencing a lot of challenges. The quality of healthcare is at an all-time low and life expectancy at birth for male/females is approximately 55/56 years (2016), one of the lowest in Africa. The probability of dying under five (per 1000 live births, 2017) is approximately 10 percent. The total expenditure on health as percentage of GDP in 2014 was 3.7 percent, significantly lower than other leading African economies (WHO, 2019).

As indicated below in Figure 1, The World Health Organization (WHO) reported that Nigeria's government and private health spending per person in 2015 was US\$97. In the same year, South Africa's was US\$471, or about five times more. This difference represents a steady fall from 2010 and 2011 when it was seven times higher. When adjusted for the cost of living, Nigeria's per capita expenditure on healthcare was PPP\$215 in 2015, while South Africa's was PPP\$1,086 - also five times higher (Okpi, 2018). 




Chart: Africa Check - Source: WHO - Get the data

Figure 1. Health spending in South Africa and Nigeria (2010-2015). Government and private spending on health per person in US dollar and also adjusted for the cost of living in each country (Purchasing Power Parity, or PPP)

Nigeria was ranked low (140th position out of 195 countries) in the Global Healthcare Ranking. The global healthcare ranking is based on a qualification of personal access and quality for 195 countries and territories from 1990 to 2015 (The Guardian, 2017).

In a study that was published by The Lancet on the Healthcare Access and Quality Index, out of the 32 diseases for which death rates were tracked, which include tuberculosis and other respiratory infections; illnesses that can be prevented with vaccines (diphtheria, whooping cough, tetanus and measles); and several forms of treatable cancer and heart disease and maternal and neonatal disorders, Nigeria lagged far behind most other African countries. The result of The Lancet study shows that while globally most countries improved over the period of observation, inequalities have increased in some parts of the world, and in particular, in many low-to-middle income countries and in subnational geographical areas of several countries like China, India, Brazil, Mexico, other Latin America countries, and numerous African nations (The Guardian, 2017).

The state of healthcare delivery in Nigeria seems to be appalling and there has been too much national lamentation for too long concerning the country's poor health infrastructure, poor remuneration for doctors, inadequate administration, and insufficient government's funding. Nigeria has some of the best medical doctors and health practitioners in the world, but unfortunately, it seems that most of those highly educated and talented medical professionals are out in foreign countries excelling where the conditions and atmosphere are more favorable. Nigerians, who can afford it, continue to flock to foreign countries to seek medical help while the poor are left with no option than to seek for the low-quality healthcare services provided at national level (The Guardian, 2017).

In spite of the availability in the country of Health Insurance Schemes such as, National Health Insurance Scheme (NHIS), National Immunization Coverage Scheme (NICS), Midwives Service Scheme (MSS), and Nigerian Pay for Performance Scheme (P4P), it seems that currently over 90 percent of the Nigerian population is without an health insurance coverage, which forces many households and individuals to bear the burden of a dysfunctional and inequitable health system, causing them either to postpone healthcare cures or not to seek at all healthcare services having to pay out of pocket for those services which are not affordable. Poor people constitute about 70 percent of the Nigerian population. They lack access to basic health services because they cannot afford it (Aregbeshola, 2019).

According to Nigeria Demographic Health Survey (NDHS) of 2013, over $60 \%$ of pregnant women aged $15-49$ years deliver their babies at home without any antenatal care visits. In rural areas, this value reaches $76.9 \%$. The situation is critical in North East and North West regions of Nigeria where over $79 \%$ of pregnant women aged 15-49 years deliver their babies at home. The distance of pregnant women's homes from a health facility and the cost of healthcare are some of the reasons for not delivering at a health facility. The cost of healthcare and perceived low quality of care by the public have been argued to be the reason for the poor utilization of maternal and child health services in Nigeria (Aregbeshola, 2019).

In summary, among the major problems with the healthcare sector of Nigeria the following ones typically seem to top the list: high cost of healthcare; poor funding; shortage of skilled manpower; widespread inequality, and poor access to healthcare in rural areas and remote communities. Telehealth and Mobile Healthcare devices can help alleviate at least some of these pressures, as well as, extend medical services to underserved or unreached areas. There seem to be an urgent need for innovative business models and infrastructures that might help disrupt 
the industry, making healthcare more affordable and accessible for most people.

This paper highlights the importance of technology-driven innovation and the exciting new opportunities that global advancements in digital medicine may provide to Nigeria, and other developing nations, through the acceleration of the urgent and much-needed transformation of the healthcare system. This paper therefore seeks to examine ways in which the healthcare industry can be disrupted in Nigeria. The study is based on multiple data sources and on the valuable answers to an online survey provided by a selected sample of highly educated and experienced Nigerian professionals during a period of three months (March-May 2019). The results of the survey are quite encouraging and unveil an optimistic scenario about the potential successful diffusion of Mobile Health and Telehealth services in the country in the near future (circa 5 years).

\section{Literature Review}

\subsection{What is Disruptive Innovation?}

The term disruptive innovation is frequently associated with the theoretical framework introduced by Clayton Christensen in 1995 which has been widely referred to as one of the most influential business ideas of the 21 st century. Christensen defined disruptive innovation as an innovation that targets a new market or the low-end of an existing market and that creates a new value network. After gaining a foothold in the target market, the disruptor's strategy eventually grows and improves over time (typically, but not exclusively, also through a process of technological upgrading) until it eventually displaces the strategy and competitive positioning of well-established firms in the industry (Christensen et al., 2015).

\subsection{What Is Sustaining Innovation?}

Sustaining Innovation is an improvement in the performance and profit margins of established products that mainstream customers in major markets have historically valued. It uses its existing business model, value networks, culture, and capabilities to continue to offer new and improved products to its most demanding and strategic customers. Sustaining innovation can be either incremental or radical (Christensen et al., 2015).

In today's era of digital revolution and 4.0 technology transformation, always more product categories and industries are constantly disrupted thanks to quick, agile, lean, convenient, and groundbreaking innovations and strategies. For example, tablets, latest mobile devices, app-based services, and advanced technological solutions based on big data, machine learning, deep learning, IoT, virtual and augmented reality, cognitive computing systems, multisensory interfaces, and AI-based technologies are completely redefining the perimeter of entire industries and are increasing the disintermediation of distribution channels. These business models often lead to industry convergence, disrupt industries' incumbents, and create new dimensions of customer experiences, customer expectations, and innovative interactions with customers and value networks through social media, communities of experts, open innovation models, and digital transformation models. In the mobile technology era, smartphones and tablets for example are becoming devices of disruption of traditional personal computers and traditional firms' business models based on the "brick and mortar" distribution of products and services. The traditional concept of product ownership is being redefined by a growing attention of consumers to the benefits of "access and use" of shared resources versus the benefits of ownership; by their preferences for companies that pursue also a social purpose besides shareholders' profit maximization; by a rising attention to sustainability goals and the ecological impact of resource utilization; by the need for more customized solutions; and by new ways of shopping and experiencing products' consumption thanks to digital and mobile technologies, which allow them to share in real-time digital and physical in-store experiences with other members of their digital community and with the surrounding environment (i.e., IoT devices, multisensory interfaces, and AI-based technologies, smart cities' interfaces) (Pezzuto, 2019b). 




Figure 2. Disruptive and sustaining innovation

Source: Christensen et al., 2015.

Figure 2 shows the typical innovation trajectories of disruptive versus sustaining innovation strategies over time and the competitive edge of disruptors who offer a better fit value proposition and value network (i.e., good-enough) for customer segments that are overshot by the industry incumbents' value propositions.

\subsection{Disruptive Versus Sustaining Innovation in Healthcare}

Disruptive Innovation has erupted in almost all industries including media, telecom, finance, and retail. New technologies, new business models and competition have given rise to more affordable, easy-to-use, and accessible products and to disintermediation in the distribution channels. This has not been the case for many years in the healthcare industry globally until recently. As technology advances in healthcare, there has been in many countries a steady rise in the cost of healthcare. Traditional players in the healthcare industry have been engaging mostly in sustaining innovation, investing more in making more sophisticated and expensive products for their best customers, although in recent times major technological developments and disruptive business models have emerged in the USA, China, and other leading nations, driven by the experimentation of highly innovative startups and giant tech companies.

Healthcare has therefore become quite expensive and not easily accessible in many geographical areas of the world and thus it is in urgent need of disruptive innovation and is said to be ripe for disruption. According to Christensen et al. (Christensen, 2017), high costs and uneven levels of access are typical hallmarks for an industry that is ripe for disruption.

A truly disruptive innovation initially targets the low-end and marginal segments of the market or new markets which are typically overlooked by the industry incumbents. The value innovation of the incumbents (i.e., sustaining innovation) typically overshoots the needs and purchasing power of these segments. After gaining a strong foothold in these segments of the market, delivering to them a more-suitable functionality-frequently at a lower price, the disruptor relentlessly moves up market, eventually displacing established competitors and changing dramatically the rules of the game in the marketplace. Often, the disruptive innovation comes with a technological shift that simplifies consumption for users and makes the value proposition more convenient, accessible, and affordable for the underserved segments (Pezzuto, 2018a).

Disruptive innovations are powerful means for broadening and developing new markets providing new functionalities, which will eventually disrupt existing market segments and linkages (Adner, 2006; Charitou \& Markides, 2003; Christensen, 1997; Bower \& Christensen, 1995; Christensen \& Raynor, 2003; Govindarajan \& Kopalle, 2006; Yu \& Hang, 2010). In Figure 3, here below, is summarized the timeline of evolution of the Disruptive Innovation Theory. 




Figure 3. Timeline of evolution of disruptive innovation theory (Yu \& Hang, 2010)

Source: Yu \& Hang, 2010.

\subsection{Creating Shared Value (CSV) Strategies.}

Scholars such as, Michael Porter and Mark Kramer $(2011,2018)$, argue that there seem to be an urgent need for business to rethink its role in society by incorporating a social purpose into core competitive strategy, and this is particularly true for emerging and developing markets. For this scope, these scholars have proposed the concept of Creating Shared Value (CSV), which is an innovative "approach that treats social issues as a source of economic opportunity and competitive differentiation for business, rather than a social obligation or cost of doing business" (Porter \& Kramer, 2011; 2018). Thus, ultimately, the authors encourage forward-thinking firms to embrace the CVS approach since it is a source of competitive advantage that creates measurable social impact for society at large scale and economic benefits for the business, "unlocking the next wave of business innovation and growth" (Porter \& Kramer, 2011; 2018). The healthcare industry in Africa seems to be an ideal context for implementing successful CSV strategies through the adoption of disruptive business models that might improve patients' access to affordable healthcare services, support healthcare industry sustainability, and help innovative firms achieve value creation goals and foster social progress.

\section{Method}

The research methodology applied for this research is based on an exploratory (qualitative) study which aims to provide answers to the following research questions:

1) Can Mobile Health devices and Telemedicine services (i.e. Remote Patient Monitoring) be a potential opportunity for providing/improving access to an "acceptable level" of healthcare services for the poorer and underserved or underreached patients in Nigeria?

2) Are innovative Mobile and Telehealth services expected to be introduced in Nigeria in the near future to serve the poorer and underserved or underreached segments of the market?

3) Are the available technological infrastructures in Nigeria able to support the delivery of good quality Mobile Healthcare services and Telemedicine services (i.e. Remote Patient Monitoring) to the poorer and underserved or underreached segments of the market?

4) Will the introduction of these innovative technologies in Nigeria help reduce public healthcare costs and make the healthcare system more efficient and sustainable?

5) Can the availability of these innovative Mobile and Telehealth technologies help lower-income families and 
underserved or underreached patients access good quality healthcare services at more affordable costs?

6) Will these innovative technologies and Mobile and Telehealth services help improve general health conditions of the lower-income individuals and underserved and/or underreached patients in Nigeria?

7) Can existing innovative companies or innovative start-ups offer these disruptive Mobile and Telehealth services in Nigeria at affordable prices developing a scalable and profitable business model balancing corporate goals on growth and profitability with affordable, accessible, low-cost, efficient, and good quality mobile and telehealth services for the poorer, underserved, and/or underreached patients?

8) What cures, tests, disease treatments are more likely to be successfully offered in Nigeria through the use of innovative Mobile and Telehealth services?

9) Which Telehealth or Mobile healthcare devices are more likely to be introduced in Nigeria in the next few years?

10) What percentage of the lower-income, underserved, and/or underreached Nigerian population is likely to be reached and served by the Mobile Healthcare and Telehealth services in the next 3 to 5 years?

The exploratory research methodology applied in this study combines secondary data sourced from websites' content, press articles, and scientific and academic publications with primary data sourced from an online survey. A single case study research design has been used in order to offer in-depth contextual insights (in real-life contexts) on the topic of research (the investigated phenomena), and on its specific research questions, taking into consideration environment characteristics, resource constraints, and economic and cultural traits of the country.

In fact, as suggested by Yin (2018), the choice of a single case study can be appropriate in order to explore and gain insight on new, innovative, and more complex issues within the context of real life by analyzing a small number of events or conditions through pattern matching, rather than theory testing (Yin, 2018).

\subsection{Qualitative Research Design}

Given the explorative and forward-looking nature of the research, a semi-structured online questionnaire has been created for this study on Survey Monkey consisting of 10 closed-end questions, which rely on the use of Likert scales ( 1 to 5$)$ for the answers, and 10 open-ended questions, which allow the responders to provide a deeper and more insightful answer for each closed-ended research question. Clear guidelines have been provided in the online survey to clarify the "target profile of participants" eligible to participate to the study and the scope of the explorative research.

\subsection{Participant Characteristics}

Nigerian participants to the online survey have been sought based on their professional expertise; higher education background, and their profiles' good fit with the topics of the research questions. A purposeful (Judgmental) sampling process has been used to select the participants to the study in order to gain valuable forward-looking insights from them on the Nigerian business and technological environment, culture, and entrepreneurial readiness for disruptive innovation opportunities in the healthcare industry.

\subsection{Sampling Procedures and Sample Size}

The choice of a purposeful (Judgmental) sampling process is coherent with the scope of the explorative and forward-looking qualitative nature of the research. The selected sample consists of forty highly educated (most of them hold master and doctoral degrees) and qualified Nigerian professionals and experts whose average age is approximately forty-four years old. The participants to the online survey have provided their answers in the period March-May 2019, which have been collected and analyzed by the author of this paper towards the end of May 2019 for the write-up of the research findings. Data validation has been facilitated through cross verification combining several methods typically used in the same phenomenon studied.

\section{Environmental Analysis of Nigeria, Healthcare Industry Challenges, and Disruptive Innovation Opportunities}

Nigeria is a so-called "emerging market" of the African continent which has experienced in the last decade a rapid population growth, rapid urbanization, growth in the middle class, increasing foreign direct investments (FDIs) and a relatively stable political environment. Nigeria has been identified as a regional power in Africa, with one of the strongest economies in the region, and it is expected to become one of the world's top 20 economies by 2050 (World Bank, 2016).

Nigeria is a promising economy, although currently (early 2019), it is in the middle of a transition phase of 
slower economic growth. In fact, as indicated in Figure 4 here below, Africa's most populated country with approximately 190 million of inhabitants $(51.0 \%$ of the population is urban and the median age in Nigeria is 17.9 years), according to recent demographic trends projections, is expected to become the third most populous country in the world by 2050 (IMF, 2018). Yet, it is still facing a number of social, economic, institutional, and political challenges.

\section{Burgeoning population \\ Nigeria could have the world's third largest population by 2050.}



Source: United Nations Population Statistics.

Figure 4. Nigeria could have the world's third largest population by 2050

Source: United Nations Population Statistics, 2017.

The following Figure 5 (PEST analysis) provides a summary review of Nigeria's macro-environment. 




Source: Pezzuto 2019; Osagie, 2019.


Figure 5. PEST analysis

\subsection{Latest Development, Issues, and Challenges in the Health Industry in Nigeria}

Life expectancy in Nigeria is one of the lowest in Africa based on WHO statistics of 2016 (World Health Organization, 2019) due to a relatively weak health system. Nigeria is faced with a wide range of economic, health, security and political challenges and as such healthcare struggles to emerge on the government's top priority agenda (Alloh \& Regmi, 2017; Adenuga et al., 2017; Adeloye et al., 2017).

Nigeria has five hospital beds per 10,000 population. The Federal Ministry of Health's (FMOH) health facilities (HFs) census of 2005 showed that Nigeria had a total of 23,640 public and private hospitals (PharmAccess Group, 2015). The densities of nurses, midwives and doctors in the country are still too low to effectively deliver essential health services $(1.95$ per 1,000$)$. The health workforce is concentrated in urban tertiary health care services delivery in the southern part of the country, particularly in Lagos (GHWA, 2019).

Michael Olarewaju, the president of the Association of Resident Doctors Federal Capital Territory Administration, Abuja chapter, stated that on average about 12 of his colleagues leave Nigeria every week to work overseas. He also stated that less than 40,000 registered doctors are currently practising in the country of about 190 million population. He lamented that not only the majority of Nigerians lack access to quality health care, over 88 per cent of doctors in the country desire to leave for greener pastures overseas, but he mentioned that among major reasons behind the mass exodus of doctors from Nigeria to countries such as, United States, United Kingdom, Canada, Australia, South Africa and the United Arab Emirates there are also poor pay, lack of career advancement opportunities and job insecurity (Adebowale, 2018).

\subsection{The Disruptive Innovation Opportunity of Telemedicine and Mobile Health Systems}

It is widely accepted that innovation is key to the transformation of the healthcare sector. Innovation will contribute significantly to lowering cost, growth, and improving the quality of healthcare delivery. Disruptive innovation might offer effective, low-cost solutions that would be easily accessible and simple to use targeting the low-end of the market. New technological innovations in healthcare can offer huge opportunities for transforming lives but these technologies must be low-cost in order to be feasible and implementable in developing nations like Nigeria.

As explained by Siddique Latif et al. (2017), Telehealth and mHealth (Mobile Health) systems allow physicians to easily access the health-related medical information anytime, anywhere, and to take major decisions remotely on the clinical care of patients in distant areas. The more technologically advanced and affordable mobile telephony standards (i.e., $3 \mathrm{G}$ and $4 \mathrm{G}$, and more recently $5 \mathrm{G}$ ) and cloud-based mHealth systems become, the 
easier will be to reach and serve mHealth services to the poorest people in the developing countries. Here below Figure 6 reports a typical mHealth architecture in which data from various mobile devices pass through a network of remote servers in order to be accessible by healthcare practitioners (Latif et al., 2017).



Figure 6. Typical mHealth architecture

Source: Latif et al. (2017). Mobile Health in the Developing World: Review of Literature and Lessons from a Case Study, IEEEE Access.

Telehealth supports the delivery of healthcare, public health, and health education services at a distance, as well as, provides a collaboratory platforms for sharing knowledge and expertise and engaging communities in participatory research. Figure 7 below gives a further breakdown of the term 'Telemedicine'.



Figure 7. Telehealth and its components

Source: Ingenium Telehealth Business Plan, 2017.

Telemedicine can be a workable solution to the shortfall in skilled medical specialists and limited access to quality healthcare in rural areas through provision of services such as digital health, video consultations, remote patient monitoring, which can also be integrated by the use of Small Ultrasound Scanners and drones. The rapid 
advancement in telecommunications on the African continent has opened up avenues for improving medical care to underserved populations (Sarfo et al., 2017; Adenuga et al., 2017).

It seems to be a quite likely future scenario the rapid expansion of virtual health and telemedicine 4.0, although it is forecasted that self-service Telehealth apps incorporating artificial intelligence (AI) into the process will remain mostly hybrid models, involving some form of human interaction (Roth, 2018).

\subsection{The Four Delivery Modalities of Telemedicine and Mobile Health Systems}

Telehealth and mHealth encompass four distinct service modalities.

1) Store-and-Forward;

2) Live and Interactive Video;

3) Remote Patient Monitoring;

4) Patient Engagement Mobile Apps

1) Store-and-Forward (asynchronous) involves the transmission of multimedia medical data from one provider to another for clinical evaluation outside of a real-time interaction.

2) Live and Interactive Video (synchronous) involves two-way live audiovisual interaction between a consumer and provider by computer, phone, or home health monitoring devices.

3) Remote Patient Monitoring involves transfer of personal health and medical data from a community setting to a remote provider for monitoring and providing related support.

4) Patient Engagement Mobile Apps involve the use of wearable sensors, smartphone apps, and other mobile monitoring and communication devices to provide consumers with interactive care education and outreach services (TTRN, 2017).

\subsection{Future Outlook in Telehealth and mHealth}

Analysts have predicted further disruption in the global health sector, based on rapid technological advancements such as big data, artificial intelligence etc. For example, heightened awareness on the 'big data' approach to collection and analysis of health data across world countries is expected to further bridge the gap between healthcare delivery, policy response and population health outcomes across cities, countries and continents (Wyber et al., 2015; Adeloye et al., 2016). It is expected that increase exchange of high-quality data and information within the health sector will further enhance the use of RPM (Remote Patient Monitoring) and other digital and mobile solutions and ultimately will enhance overall patients' care and improve health worker's efficiency in both low-and middle-class income countries.

The distributed ledger methodology known as blockchain is piquing interest in the healthcare industry as organizations search for more secure and trusted strategies for managing big data and mitigating potential cybercrime threats. Current uses of blockchain include the creation of a truly longitudinal health record controlled entirely by the individual and a more robust patient matching and identification system that improves patient safety. As awareness and research continue to grow, more uses of blockchain are expected to be developed, leading to better exchange capacities between hospitals, providers and payers (Mettler, 2016; Bresnick, 2017).

Blockchain and its distributed ledger technology may give patients more control of their data; improve reliability, security and accuracy of data recording, and help reduce costs. Blockchain-based electronic Health systems may create significant improvements in medical records management (Pezzuto, 2019b).

Mshali et al. (2018) highlighted the role of machine learning in enhancing the efficiency of sensor data analysis in health monitoring activities. Machine learning is also giving rise to more sophisticated image diagnostic tools, helping physicians make more accurate diagnosis and treatment recommendations.

Other health applications that are gaining momentum are: personalized medicine and automatic recommendations; health recommendations and disease treatments which are tailored based on the patients' medical history, genetic lineage, past conditions, diet, and stress levels. Also, on the rise are proactive disease prevention, performance improvement and autonomous robotic surgeries (Fagella, 2018).

Additional valuable examples of innovative solutions for Remote Patient-Monitoring devices, mHealth, and Telemedicine are those offered by the Giant Chinese Tech Company Tencent, which may also benefit from the firm's groundbreaking innovations in the field of AI-powered medical technologies. China's leading technology-enabled healthcare solutions platform, We Doctor Holdings Limited ("WeDoctor"), also provides to 
their clients seamless online and offline healthcare services (WeDoctor Healthcare, WeDoctor Cloud, WeDoctor Insurance, and WeDoctor Pharma). Interesting innovative solutions are also those offered by China-based medical app maker Quyi; those offered by the insurance company Ping An Insurance with Samsung; the ones offered by CliniCloud's smartphone-connected medical kits; Circle Medical's primary care house call app; and the AI-powered mobile assistant digital tools of Google-owned artificial intelligence company, DeepMind Health The latter one allows doctors and nurses to timely pick up patients' illness warning signs and to act on in time, through the use of predictive algorithms. Other relevant examples include: the services provided by Telehealth provider Doctor on Demand and the Intelligent hybrid Remote Patient-Monitoring model with cloud-based framework for knowledge discovery proposed by Mohammed K. Hassan; Ali I. El Desouky, Sally M. Elghamrawy, and Amany M. Sarhanc (2018). Furthermore, interesting innovations in this domain are also those provided by British telehealth startup Babylon Health aimed to power Samsung's pre-installed Health app in its new smartphones; also those provided by British diagnostic devices start-up, Concepta Diagnostics Ltd, and the innovative telemedicine services provided by Clalit in Israel. Still, other relevant examples of successful applications of technological innovations in emerging and developing economies include the use of Zipline drones (the drones of Zipline, a medical drone manufacturing company) in Rwanda for the delivery of medical supplies to local hospitals.; GE Healthcare's ultra-portable, battery-operated electrocardiogram machines specially designed for use in rural clinics in India; Mashavu's Project of Telemedical kiosks for remote diagnosis in Kenia and Tanzania, and the innovative technological devices designed to help speed up tests in Botswana for children's cures (i.e., for diarrhea) (The Medical Futurist, 2019)(Pezzuto, 2019b).

In the African continent, recently, have been introduced innovative medical devices, aimed at bringing medical imaging services to remote communities which include a battery-powered portable small ultrasound scanner that is plugged-in to mobile phones to scan patients' organs, allowing doctors, even in a distant location (i.e., iPhones make it easy to upload scans to the internet), to immediately provide a double-check of the diagnosis and prescribe a cure for the patient's illness at early-stage of the disease; and to provide a more efficient illness prevention protocol, and the containment of potential epidemic outbreaks (McNeil Jr., 2019).

In Nigeria, a number of incubators and accelerator programs have been recently launched in order to support local start-ups in their entrepreneurial ventures. There seem to be huge untapped market potential in this country's healthcare sector for innovative firms with the "right" vision, value network, and disruptive business model. Some of the most dynamic local start-ups which are highly engaged in digital health include the following: Safermom, Mobicure, Ubenwa, Medsaf, GenRx, Medical device as a service (MDAAS), Kangpe, Hudibia, Lifebank, Medismart Inc., Curacel Health, Apmis, Avon HMO, and Flying doctors Nigeria (FDN) (Hempel, 2018).

In advanced economies such as the USA, telemedicine services and mHealth services are currently exploring new technological solutions which consist of applications based on the combined use of artificial intelligence, apps, and chatbots, and videos. This innovative approach is often referred to with the expression "Virtual Health," as in the case of the Phoenix-based firm, Banner Health. Other firms using telemedicine solutions in their platforms include: American Well, Teladoc, Athenahealth, Cerner, and Epic. Telehealth apps not only guide patients through a series of questions and collect data, perhaps through a bot, but some can interpret those responses. This process makes the clinical interaction more efficient and more focused. Some of the firms involved in these business models include: Bright.MD, CirrusMD, HealthTap, 98point6, Sherpaa, and Zipnosis, Babylon, and CloudMedx (Roth, 2018).

Other dynamic innovative start-ups eager to unleash untapped growth market opportunities in the digital health space include also Clover Health, Beam Therapeutics, Health Catalyst, Hims, LinkDoc, Doctolib, Sophia Genetics, Taimei Medical Technology, Sopris Health, Parsley Health, FundamentalvR, Empiric Health, RDMD, Deep Lens Inc., Hero, Pilleve, VitaScan, Verge Genomics, HealX, Empleema, Prellis Biologics, HealthCrowd, Maven Clinic, Beddr, Heranos, uBiome, Nurx, eClinicalWorks, Practice Fusion, Tempus, and PathAI.

Recent experimentations have been undertaken in the USA by AI researchers and medical professionals at Stanford University and Intermountain LDS Hospital in Salt Lake City, Utah, which consist of using machine vision to continuously monitor patients and depth sensors to be installed in individual patient rooms in order to collected three-dimensional silhouette data for 24 hours a day over the course of two months. A final algorithm is then used for detecting mobility activities of the patients (Yeung et al., 2019).

From these few examples, it's clear that there is a growing interest and a strong drive for advancements in digital medicine globally, and in particular in emerging and developing markets, and mostly in Africa. It is believed that there will be increased transfer of these new technologies into developing nations in the future as a major driver 
for inclusion in healthcare and that investments in digital healthcare in these countries are likely to continue to rise in the years to come.

\section{Results}

Here below in Figure 8 are reported the occupational profiles of the survey's responders.



Figure 8. Occupational profile of the participants to the study (in percentage)

\subsection{Research Findings of the Exploratory Study}

The analysis of the responses to the survey reveals that a disruptive business model based on Telehealth and/or mHealth is expected to be an optimal solution to help patients monitor and manage their health conditions and to help prevent chronic illnesses, while also avoiding costly medical treatments. Here below are reported the figures and research findings related to the 10 research questions of the exploratory study:

Question 1. As indicated in Figure 9, the majority of responders (95.5\%) of the sample (i.e., $44.4 \%$ answered "Agree" and 51.1\% answered "Strongly Agree") perceive Mobile Health devices/Telemedicine as a very attractive potential opportunity for providing/improving the access for the poorer and underserved or underreached patients in the Country to healthcare services. The favorable answer to the question of the survey reveals that the perceived benefits of the Mobile Health devices/Telemedicine are broadly shared by almost the totality of the participants to the survey, regardless of their professional expertise and geographical location in the country, although it is quite obvious that many participants are based in major urban areas (Pezzuto, 2019a).

\section{Q1 - Can mHealth/Telehealth help improve access to health care services for lower- income/poor population?}

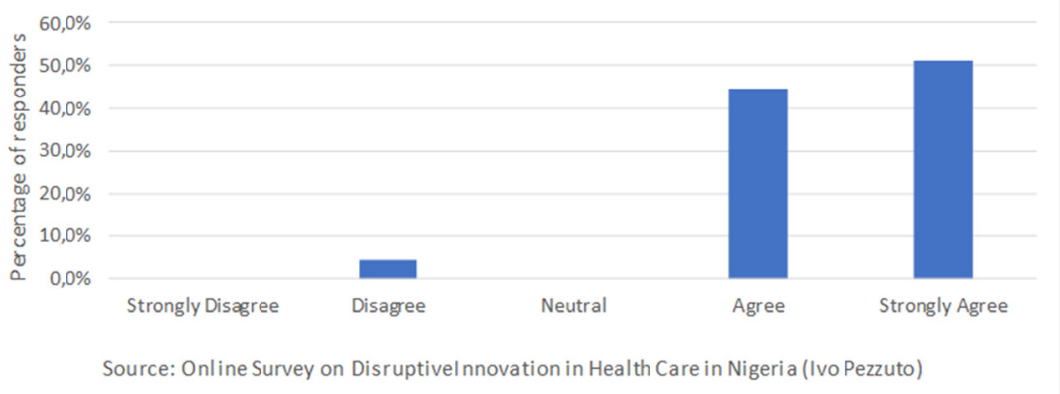

Figure 9. Q1 of the online survey on disruptive healthcare innovation in Nigeria 
Question 2. As indicated in Figure 10, approximately $70 \%$ of the responders expect Mobile Health devices/Telemedicine to be introduced in the country in the near future (within 5 years) to serve the poorer and underreached patients, but only for approximately $27 \%$ of the total responders these services will be available within the next two years. $30 \%$ of the total responders expect the availability of these services within 3 to 4 years. $13 \%$ of the total responders expect the availability of these services in 5 years. $18 \%$ of the responders do not expect the availability of these services in the near future. $12 \%$ of the responders have expressed a neutral opinion on this question (Pezzuto, 2019a).

Entrepreneur, Peter Bankole, responded to this question with the following comment: "Internet penetration is increasing very rapidly and becoming cheaper. The majority of access to the internet is via mobile phones, hence mobile devices and apps will play significant role in this evolution. We have already seen such innovation in the financial services sector, retail, logistics, etc. in Nigeria. These services will certainly reduce public healthcare costs as basic primary healthcare will now be more accessible to the vast majority of the people. It will also bring about the creation of new jobs and "digital" rural economy will begin to evolve. Rural-Urban migration will slow down as young persons can now be "healthcare agents" in addition to using the same infrastructure to do other things such as fintech, etc." (Pezzuto, 2019a).

Medical doctor, Edward Carpe, has provided the following comment to this question: "With the steady improvement with power sources and the availability of alternative sources of power e.g., solar, I expect that in the next five years telemedicine and mHealth would be more commonplace in Nigeria. Drones too will be more available in delivering medications and also other life-saving materials in remote areas. Portable devices like ultrasound scanners and others will be more available too" (Pezzuto, 2019a).

\section{Q2 - Are mHealth/Telehealth services expected to be introduced in the near future?}



Figure 10. Q2 of the online survey on disruptive healthcare innovation in Nigeria

Question 3. As indicated in Figure 11, approximately 90\% of the responders declares that the country's technological infrastructures will be able to support in the medium-term (within 5 years) the effective delivery of Mobile Health/Telehealth services. A more granular analysis of the responders' answers, however, reveals that only approximately $15 \%$ of the total responders believe that the existing infrastructures can enable the immediate implementation of these services. $25 \%$ of the responders believe that it will take at least 1 to 2 years before the infrastructures will ready; $30 \%$ of the responders believe that it will take about 3 to 4 years; whereas about $20 \%$ of the responders believe that 5 years will be necessary to have the enabling infrastructures in place (Pezzuto, 2019a).

Medical doctor, Edward Carpe, has provided the following comment to this question: "at the moment there is still an infrastructure gap in some areas. This is because there are still many places in the creeks of the Niger Delta and remote parts of the North where these services may be difficult to provide with the present level of infrastructure. However, in the semi-urban and urban areas, this is very possible even at this moment" (Pezzuto, 2019a). 


\section{Q3 - Can technological infrastructures enable the delivery of $\mathrm{mHea}$ th/Telehealth services?}



Figure 11. Q3 of the online survey on disruptive healthcare innovation in Nigeria

Question 4. As indicated in Figure 12, approximately 84 percent of the responders (i.e., 37.4\% answered "Agree" and $46.6 \%$ answered "Strongly Agree") believe that the availability in the country of Mobile Health services/Telemedicine services would help reduce the public health care costs and would help make the health care system more sustainable in the medium-term (within 5 years). A more in-depth analysis of the data indicates, however, that only approximately $27 \%$ of the total responders expect that delivery of these services will generate immediate benefits to patients and the health care system. $24 \%$ of the total responders believe that it will take at least 1 to 2 years to see the benefits in costs reduction and improvement of the healthcare system sustainability. $20 \%$ of the total responders instead reported that it would take at least 3 to 4 years to see the benefits. $13 \%$ of the total responders reported that 5 years will be necessary to see the benefits on costs and sustainability (Pezzuto, 2019a).

\section{Q4 - Will mHealth/Telehealth services reduce public healthcare costs/improve sustainability?}



Figure 12. Q4 of the online survey on disruptive healthcare innovation in Nigeria

Question 5. As indicated in Figure 13, approximately $82 \%$ of the responders (i.e., 25.3\% answered "Agree" and $56.7 \%$ answered "Strongly Agree") believe that the availability of Mobile Health/Telemedicine services in the medium-term (within 5 years) may provide access to healthcare services at more affordable costs for lower-income families. A relatively small percentage of the total responders (8\%) has reported to disagree with the hypothesis of more affordable healthcare services (i.e., $6 \%$ answered "Disagree" and $2 \%$ answered "Strongly Disagree"). $31 \%$ of the total responders reported to expect immediate reductions to healthcare costs. $16 \%$ of the participants to the survey expect more affordable healthcare costs after 1 to 2 years; $18 \%$ expect lower costs after 3 to 4 years; $17 \%$ expect lower costs in 5 years (Pezzuto, 2019a). 
Regarding this specific question, Public Health Consultant Gbenga Olayiwole, has explained the following concept: "With the expectation of early detection and treatment of basic illnesses, low-income families should have their expenses on treatment of basic illnesses reduced" (Pezzuto, 2019a).

Regarding this specific question, medical doctor Adeleke Oshunniyi, has reported the following concept: "The Nigerian Health Sector is underfunded. With a grossly insufficient allocation in the 2018 Federal Budget of N359 Billion and recurrent expenditure of over N270 Billion this amounts to only about N400 per person per annum. Out-of-pocket expenditure is now estimated to be over $70 \%$ of health expenditure. An increase in health funding, if well managed, will translate to better health indices." (Pezzuto, 2019a).

\section{Q5 - Can mHealth/Telehealth help make healthcare services more affordable?}



Figure 13. Q5 of the online survey on disruptive healthcare innovation in Nigeria

Question 6. As indicated in Figure 14, approximately $86 \%$ of the responders (i.e., 27.7\% answered "Agree" and 58.3\% answered "Strongly Agree") believe that the availability of Mobile Health/Telemedicine services will improve the general health conditions of the lower-income individuals and underserved or underreached patients of Nigeria in the medium-term (within 5 years). $50 \%$ of the total responders believe that the availability of these services will immediately improve the health conditions of the lower-income and underserved population of the country. $10 \%$ believe that the improvement will occur only after 1 or 2 years; $8 \%$ believe that the improvements will occur after 3 to 4 years. 18\% believe that the improvements will occur in 5 years (Pezzuto, 2019).

Medical doctor, Edward Carpe, has provided an insightful contribution to this question: "doctors working in under-served and under-reached communities can with the aid of mobile healthcare services, telemedicine, etc. make timely interventions that can save lives. For instance, they may be better able to make a diagnosis of ectopic pregnancy before the patient actually ruptures where availability of large quantity of blood becomes a critical requirement to a successful surgery. People are still traveling long distances in some parts of the country just to do a scan or get an X-ray done. This adds to the cost of healthcare. Some of these people therefore resort to traditional medicine since they cannot afford the cost of the journey combined with the actual cost of the investigation. There is also the disadvantage of not been able to make a diagnosis promptly in some instances in order to effect life saving measures such as surgeries. An example is the absence of ultrasound scanners in a case of ectopic pregnancy" (Pezzuto, 2019a).

Civil servant, Dare-Lawrence Jumoke, made the following comment on this question: "the availability of these services will boost accessibility with proper awareness. Competitive market dynamics will bring the cost down to what is affordable to the people, but there must be widespread public awareness that these services exist at affordable prices" (Pezzuto, 2019a).

Public health consultant, Gbenga Olayiwole about his question commented: "with economics of scale, costs should come down after a few years as long as deployment is effective and geographic coverage is large enough, costs will come down" (Pezzuto, 2019a). 




Figure 14. Q6 of the online survey on disruptive healthcare innovation in Nigeria

Question 7. As indicated in Figure 15, approximately $86 \%$ of the responders (i.e., $42 \%$ answered "Agree" and $44 \%$ answered "Strongly Agree") believe firms may develop a scalable and profitable business model in Nigeria in the medium-term (within 5 years) to offer Mobile Health/Telemedicine services to lower-income individuals and underserved and/or underreached patients. Only $4 \%$ of total responders reported to strongly disagree with the hypothesis of a scalable and profitable business model. $10 \%$ of the total responders reported not to be able to express a clear opinion on this specific research question. Approximately $37 \%$ of all responders reported that a telehealth would be almost immediately scalable and profitable; $17 \%$ reported that it would be profitable within 2 years. $17 \%$ reported that it would be profitable within 3 to 4 years. $15 \%$ reported that it would be profitable in 5 (Pezzuto, 2019a).

According to medical doctor, Edward Carpe, "the Nigerian market has depth and volume due to our ever-expanding population coupled with the increasing health seeking behavior of the populace, this should allow the sustainability of a profitable and scalable business model" (Pezzuto, 2019a).

Civil servant, Dare-Lawrence Jumoke, made the following comment on this question: "there are innovative businesses and startups that can offer these services in Nigeria with the right equipment, knowledge or education, personnel and financing, thus there is no doubt that a scalable and profitable business model is achievable in the coming years" (Pezzuto, 2019a).

\section{Q7 - Can firms develop a scalable and profitable mHealth/Telehealth model?}



Figure 15. Q7 of the online survey on disruptive healthcare innovation in Nigeria

Question 8. As indicated in Figure 16, overall for approximately $74 \%$ of the responders, the availability of mobile health/telehealth services is likely to reduce mortality rates or to improve diseases' diagnosis and treatments for the patients in the medium-term (within 5 years). Mobile/Telehealth services are expected to have 
the highest impact in the potential reduction of children's mortality rates and maternal deaths (about $80 \%$ of responders expressed this opinion), and in the speed up of the tests for children and adults' cures (respectively about $77 \%$ and $75 \%$ of responders expressed this opinion). Slightly lower chances of success have been assigned instead to stroke and heart diseases (69\%); reduction of measles, whooping cough, and diphtheria (71\%). Only $10 \%$ of the responders have reported to disagree with the hypothesis of potential improvements. $16 \%$ of total responders expressed a neutral opinion on the matter (Pezzuto, 2019a).



Figure 16. Q8 of the online survey on disruptive healthcare innovation in Nigeria

Question 9. As indicated in Figure 17, the answers of the responders to Q9 of the survey reveal that the mobile/telehealth services which are expected to have the highest probability of success in the medium-term (within 5 years) are the following: Patient Engagement Mobile Apps (84.3\%); Store-and-Forward (asynchronous) services (78.4\%), and Remote Patient Monitoring and Live and Interactive Video (synchronous) services (76.5\%) (Pezzuto, 2019a).



Figure 17. Q9 of the online survey on disruptive healthcare innovation in Nigeria 
Question 10. As indicated in Figure 18, the answers to Q10 of the survey show that for approximately $32 \%$ of the total responders $20 \%-39 \%$ of the lower-income population can be reached and served in the medium term (within 5 years). Lower percentages of responders believe instead that Mobile/Telehealth services would reach and serve more than $50 \%$ of the lower-income population and/or those located in rural areas. The majority of the responders indicated that the most likely percentage of lower-income population served would be $20 \%-29 \%$ (Pezzuto, 2019a).

\section{Q10 - What percentage of the lower-income population is likely to be served by mHealth/Telehealth in the next 3 to 5 years?}



Figure 18. Q10 of the online survey on disruptive healthcare innovation in Nigeria

\subsection{Key Insights from the Survey Responders}

Below are summarized the most important elements for the successful implementation of a Mobile/Telehealth business model in Nigeria according to the responses provided by the participants to the open-ended questions (in-depth analyses) of the survey.

\section{a) Personalized Health Coaching}

A number of responders to the study (circa 40\%) have also pointed out the critical importance of Personalized Health Coaching services for the successful diffusion of mobile and telehealth services in Nigeria. Training and professional health coaching are considered essential to facilitate awareness, dialogue, and effective implementation of these services. The coaching support is particularly important for rural areas of Nigeria were people are mostly uneducated and thus they may experience difficulties accessing telemedicine or mHealth services (Pezzuto, 2019a).

This idea was first promoted by Dr. Rushika Fernandopulle, the founder of Boston-based Iora Health, and it involves deploying trained health coaches that would offer primary care services that doctors would not normally offer such as, one-to-one continuous dialogue, checkups, follow-up reminders, and so on (Beckman \& Gupta, 2018). The main value proposition will be based on proximity to the patient, close supervision, regular one-to-one care attentions, empathy, and on the use of mobile or remote patient monitoring devices.

\section{b) Community Cooperation and Device Sharing}

Many responders (circa 60\%) to the study have also pointed out the importance of the role of community cooperation. The Community Cooperation and Sharing Model would mainly target low-income earners in small urban or rural communities. Cooperatives can be set up in order to ease the financial burden of acquiring the monitoring devices. Community cooperative members can make small monthly contributions that may entitle them to share a set of Mobile/Telehealth systems with cooperative members (Pezzuto, 2019a).

\section{c) Digital Financing, Crowdsourcing/Crowdfunding, and Mobile Money Solutions}

Many responders to the study (circa 50\%) have also stressed the importance of leveraging on the great 
opportunity of advancements in financial innovation and financial inclusion in the country, and on the benefits of the efficiency of mobile payment devices. Considering the fact that the payments will be periodic and the target market will be low-income areas, which have a high percentage of persons who are excluded from the banking and financing sector, many responders claimed that a mobile payment platform would be critical for the success of the business model. Collection of revenue from subscriptions and services has to be enabled by a simple and easily accessible platform like mobile money (Pezzuto, 2019a).

Recently, peer-to-peer value exchanges and payment systems, using digital wallet platforms and highly scalable payment services for low-income unbanked individuals of the emerging markets are becoming very popular and widely spread in many countries (Pezzuto, 2019a). The disruptive Telehealth model has to be profit-oriented and self-sustainable, nevertheless, especially in the early stage of the project, valuable financial support to these initiatives may even come partially from impact investing initiatives (one of the fastest-growing areas of asset management), fintech organizations, crowdfunding platforms, microfinance lending officers, venture capitalists, big pharma firms, medical charities, community contributions, and by philanthropic organizations like The Bill and Melinda Gates Foundation. Digital technology can achieve economies of scale that bring fintech and peer-to-peer lenders' overhead costs down, reduce transaction costs, and increase speed of operations. Cloud-based AI products can enable the lenders to standardize procedures and security levels in different countries relying on Blockchain's decentralized technology (Murray, 2019).

\section{d) Disruptive Health Insurance Models}

A number of responders (circa 40\%) have claimed that Home Health systems and disruptive Mobile Health models would be more effective if the health insurance develops also disruptive models. The insurance industry is not very developed in Nigeria, although it is improving, and needs to be strengthened. Most Nigerians, especially the low-income earners in rural communities do not have any insurance coverage due to the high cost of insurance packages. A primary cause of the problem is that the majority of them are in the informal sector of the economy. There is a need for the development of a simple, efficient, and low-cost insurance model that will result accessible to low-income communities. Projects of this nature can lower financial barriers for people who desire to seek medical care (Pezzuto, 2019a).

A relevant and likely viable example of sustainable and socially inclusive mHealth and Telehealth business model for Nigeria can be the one implemented by Jack Ma's Ant Financial conglomerate in China, which is part of the Alibaba Group Holding Ltd. This "crowdfunding for health care" model launched in 2018 is based on a healthcare-coverage product, namely Xiang $\mathrm{Hu} \mathrm{Bao,} \mathrm{which} \mathrm{covers} \mathrm{a} \mathrm{huge} \mathrm{customer} \mathrm{base} \mathrm{of} \mathrm{about} 65$ million clients and requires no payment upfront to join the service. Clients pay small monthly fees that are pooled to help cover treatment costs for members stricken by diseases such as cancer, Alzheimer's and even Ebola. This Chinese venture is upending the conventional health-insurance business by creating what essentially are online collectives. Customers can sign up for the services, make monthly payments, and even upload medical documents and bills within just a few clicks using smartphones' apps. The companies generate revenue by keeping a percentage of every payout, which is typically $8 \%$. Technology-enabled insurance, which includes everything from policies sold online to wearable device-linked insurance coverage can generate very profitable premiums for the company servicing a huge and rapidly scalable customer base. Furthermore, Ant Financials' artificial-intelligence software is also capable of recognizing claims and medical records after going through tens of millions of receipts allowing China's underdeveloped insurance market to enjoy double-digit growth. This disruptive Insuretech venture is currently triggering an insurance industry revolution in China and it might very likely create an insurance industry revolution also in Nigeria in the coming years (Bloomberg New, 2019).

\section{e) Power and Energy Sources}

As stated by Latif et al. (2017), battery life for Mobile Healthcare devices can be one of the major challenges for mHealth applications since these applications, due to their complexity, often consume significant battery power. Developing power-aware health applications is particularly crucial for developing countries as most of these countries do not have continuous and guaranteed power supply, hence failing to fully charge the battery overnight. (Latif et al., 2017). Many of the responders to the survey of this study $(70 \%)$, have reported that a true leapfrogging innovation opportunity in mHealth and Telehealth in Nigeria will occur when new and abundant alternative sources of power and energy will be widely available at affordable costs. Many have mentioned in their answers the critical importance of solar energy and other renewable energy sources as a possible solution to this critical component of the mHealth/Telehealth business model (Pezzuto, 2019a).

\section{f) The Importance of Rapidly Scaling-up the Business with Favorable Institutional Support}

In general, responders to the study $(80 \%)$ have remarked that Mobile health devices and Telemedicine in Africa 
has so far produced positive outcomes such as improved care, better awareness and education. Many responders to the survey commented in their answers that a strong commitment of the Nigerian Government (also to overcome bureaucratic barriers) and a strategic public-private collaboration would be probably necessary to accomplish the Telehealth and mHealth revolution and to offer affordable and easily accessible health care services to the lower-income population of the country. These projects, once they are scaled up and sustained by an appropriate institutional support, may lead to an overall strengthening of the national healthcare system; to a reduction in diseases and deaths, and to an improvement in the general wellbeing and longevity of the Nigerian population (Pezzuto, 2019a).

\section{g) The Importance of not ignoring the typical reasons why digital health start-ups fail}

A number of responders to the survey (circa 30\%) also confirmed with their answers the insightful research findings reported in a recent article by the Medical Futurist blog on the typical mistakes to avoid for the successful launch of digital health ventures (Medical futurist, 2019). In fact, the responders to the online survey reported that based on their experience and understanding of the local business environment, the main reasons for failure of the digital health start-ups are the following: (1) ignoring patients' specific preferences and needs and diversity issues; (2) failing to create also an attractive and compelling business model for the care providers (i.e. doctors, hospitals, pharmacies, paramedical staff, pharmaceutical companies, insurance companies, etc.); (3) failing to demonstrate significant clinical value creation; (4) the inability to deliver appropriate scientific evidence for a healthcare solution (scientific validation); (5) overclaiming what technologies can do for the patients; (6) failing to bring the product and digital/online services to patient's locations; (7) failing to build a sustainable and cost-effective business model and miscalculating profit margins and pricing strategies; (8) failing to take into account the real conditions of the local infrastructures and claiming to offer a user-friendly solution that in reality is not compatible with the medical facility's IT system available in the country; (9) failing to remember that the healthcare industry can be reluctant to change; (10) failing to enter the market at the right time.

\section{Conclusion}

This paper is based on a qualitative study which is exploring the opportunity of diffusion of mobile health/telehealth services in Nigeria to improve inclusion and sustainability of healthcare services for the lower-income population and those living in remote rural areas. The need for disruptive business models and the need for Africa to embrace recent advancement in digital medicine have been highlighted based on the responses of the participants to an ad hoc survey.

Research findings of this exploratory study reveal that existing business models have been so far small-scale and heavily reliant on external funding. There is need to scale up and develop sustainable business models that will ensure the long-term survival of mobile/telemedicine services in the region. To be sustainable, these business models must be supported by digital financing platforms, universal insurance packages, institutional support, and home coaching and community sharing arrangements for rural areas. These models may lead to improvements in healthcare delivery and the overall quality of life of the general population.

Research evidences of this study reveal that a disruptive mobile/telehealth model can be successful in Nigeria but mostly in the medium-term (circa 5 years or slightly more) due to a number of structural gaps; the need to develop proper funding and impact investing strategies; the need for innovative mobile payment systems, adequate and low-cost insurance programs, enabling local institutions' supports, and the need to deliver a proper educational program for the market participants and patients.

In summary, Nigeria and other emerging and developing economies may benefit from the adoption of a "leapfrogging" strategy based on the diffusion of disruptive mobile/telemedicine innovations. The research findings of this exploratory study seem to confirm that, if properly planned and executed, in the medium-term (within approximately 5 years), Nigeria might have the proper ecosystem and conditions that might allow firms with a scalable and profitable business model to offer socially inclusive and affordable healthcare services. In the long run, these firms can continue to upgrade their own business models, move upmarket, and eventually, they may even disrupt health industry incumbents.

\section{Acknowledgments}

The author of this paper would like to express his sincere gratitude to all the forty Nigerian participants who have provided their insightful and valuable contributions answering to the questions of an online survey designed for this exploratory research. They have also provided their written consent to the Data Protection Law for the use of their personal data and opinions in this paper. A table with their personal data is reported in Appendix A of 
this paper. The author would like also to thank for their excellent support during the research process two Nigerian PhD scholars of the International School of Management of Paris (Ms. Uzoezi Osagie and Ms. Lola Thompson Makinde) and Professor Daphne Halkias, a faculty member of the same Paris-based Global Business School (ISM).

\section{References}

Adebowale, N. (2018). Brain Drain: About 12 doctors leave Nigeria weekly—Resident Doctors. Premium Times. Retrieved from https://tinyurl.com/y2f9o6ur

Adeloye, D., Adigun, T., Misra, S., \& Omoregbe, N. (2017). Assessing the Coverage of E-Health Services in Sub-Saharan Africa. Methods of Information in Medicine, 56(03), 189-199. https://doi.org/10.3414/ME16-05-0012

Adenuga, K. I., Iahad, N. A., \& Miskon, S. (2017). Towards Reinforcing Telemedicine Adoption Amongst Clinicians in Nigeria. International Journal of Medical Informatics, 104, 84-96. https://doi.org/10.1016/j.jimedinf.2017.05.008

Adner, R. (2006). Match Your Innovation Strategy to Your Innovation Ecosystem. Harvard Business Review, 84(4), 98.

Alloh, F. T., \& Regmi, P. R. (2017). Effect of Economic and Security Challenges on the Nigerian Health Sector. African Health Sciences, 17(2), 591-592. https://doi.org/10.4314/ahs.v17i2.37

Aregbeshola, B. (2019). HealthCare in Nigeria: Challenges and Recommendations. Socialprotection.org. Retrieved from https://tinyurl.com/yxbpzzer

Beckman, A. L., \& Gupta, S. (2018). Redesigning Primary Care with Health Coaches: An Interview with Iora Health CEO Rushika Fernandopulle. Healthcare, 6(2), 156-157. https://doi.org/10.1016/j.hjdsi.2018.04.001

Bloomberg News. (2019, May 20). Jack Ma Is Selling Cancer Coverage for Pennies a Month in China. Bloomberg News. Retrieved from https://tinyurl.com/y5m3m975

Bower, J. L., \& Christensen, C. M. (1995). Disruptive Technologies: Catching the Wave. Harvard Business Review, 43-53.

Bresnick. (2017). Five Blockchain Use Cases for Healthcare Payers, Providers. Retrieved from https://tinyurl.com/y4nwtkbz

Charitou, C. D., \& Markides, C. C. (2003). Responses to Disruptive Strategic Innovation. MIT Sloan Management Review, 44(2), 55-63.

Christensen, C. M. (1997). The Innovator's Dilemma: When New Technologies Cause Great Firms to Fail. Boston, MA: Harvard Business School Press.

Christensen, C. M., Baumann, H., Ruggles, R., \& Sadtler, T. M. (2006). Disruptive Innovation for Social Change. Harvard Business Review, December.

Christensen, C. M. et al. (2000). Will Disruptive Innovations Cure Healthcare? Harvard Business Review, September-October.

Christensen, C. M. et al. (2004). Seeing What's Next: Using the Theories of Innovation to Predict Industry Change. Harvard Business School Press.

Christensen, C. M., \& Raynor, M. E. (2003). The Innovator's Solution: Creating and Sustaining Successful Growth (1st ed.). Harvard Business Review Press.

Christensen, C. M., Raynor, M. E., \& McDonald, R. (2015). What is Disruptive Innovation. Harvard Business Review, 93(12), 44-53.

Christensen, C., Waldeck, A., \& Fogg, R. (2017). How Disruptive Innovation Can Finally Revolutionize Healthcare. Christensen Institute. Industry Horizons, Spring 2017

Fagella, D. (2018) Machine Learning Healthcare Applications - 2018 and Beyond. Retrieved from https://www.techemergence.com/author/dan/

GHWA. (2019). Nigeria. Country Responses. WHO Global Health Workforce Alliance. Retrieved from https://www.who.int/workforcealliance/countries/nga/en/

Govindarajan, V., \& Kopalle, P. K. (2006). Disruptiveness of Innovation: Measurement and An Assessment of Reliability and Validity. Strategic Management Journal, 27(2), 189-199. https://doi.org/10.1002/smj.511 
Hassan, M. et al. (2018). Intelligent Hybrid Remote Patient-Monitoring Model with Cloud-Based Framework for knowledge Discovery. Computers and Electrical Engineering, 70, 1034-1048. https://doi.org/10.1016/j.compeleceng.2018.02.032

Hempel (2018). 15 Hottest Digital Health Start-Ups in Nigeria.Dr-Hempel-Network.com. Retrieved from https://tinyurl.com/y58hw5q8

IMF. (2018, March 15). IMF Country Focus. Nigeria: Out of Recession and Looking Beyond Oil. IMF Publications. Retrieved from https://tinyurl.com/y6nly9do

Latif, S. et al. (2017). Mobile Health in the Developing World: Review of Literature and Lessons from a Case Study. IEEE Access. https://doi.org/10.1109/ACCESS.2017.2710800

McNeil Jr., D. G. (2019). In African Villages, These Phones Become Ultrasound Scanners. The New York Times. Retrieved from https://tinyurl.com/y6nnj75u

Mettler, M. (2016, September). Blockchain Technology in Healthcare: The Revolution Starts Here (pp. 1-3). In e-Health Networking, Applications and Services (Healthcom), 2016 IEEE 18th International Conference on. IEEE. https://doi.org/10.1109/HealthCom.2016.7749510

Mshali, H., Lemlouma, T., Moloney, M., \& Magoni, D. (2018). A Survey on Health Monitoring Systems for Health Smart Homes. International Journal of Industrial Ergonomics, 66, 26-56. https://doi.org/10.1016/j.ergon.2018.02.002

Murray, S. (2019). How Developing Nations Use Tech to Reach The 'Underbanked'. Financial Times. Retrieved from https://tinyurl.com/y3qabb48

Okpi, A. (2018). Nigeria Spends 5 Times Less Per Person on Healthcare Than SA - not 7. Africacheck.org, Retrieved from https://tinyurl.com/yxq95f8a

Osagie Z. (2019) Disruptive Innovation Paper. CHS and Its Expansion into Home Health Services. International School of Management.

Pezzuto, I. (2018a). Lecturer's Notes of the Disruptive Innovation Seminar of Prof. Ivo Pezzuto at the International School of Management of Paris. ISM Paris.

Pezzuto, I. (2018b). Lecturer's Notes of the Global Economics Seminar of Prof. Ivo Pezzuto at the International School of Management of Paris. ISM Paris.

Pezzuto, I. (2019a). Online Survey on Innovation in Healthcare in Emerging and Developing Markets: The Case of Nigeria. Research activity of Professor Ivo Pezzuto. International School of Management of Paris.

Pezzuto, I. (2019b). Turning Globalization 4.0 into a Real and Sustainable Success for All Stakeholders. Journal of Governance \& Regulation, 8(1), 8-18. https://doi.org/10.22495/jgr_v8_i1_p1

PharmAccess Group. (2015). Nigerian Health Sector Market Study Report. PharmAccess Foundation. Retrieved from https://tinyurl.com/y35cmb4g

Porter, E. M., \& Kramer, R. M. (2011). Creating Shared Value. Harvard Business Review, January-February.

Porter, E. M., \& Kramer, R. M. (2018). Creating Shared Value: Competitive Advantage Through Social Impact (Module Notes for Instructors). Harvard Business School Publishing.

Roth, M. (2018). 4 Ways Telemedicine is Changing Healthcare. Healthleadersmedia.com. Retrieved from https://tinyurl.com/yyp7ujnx

Sarfo, F. S., Adamu, S., Awuah, D., \& Ovbiagele, B. (2017). Tele-Neurology in Sub-Saharan Africa: A Systematic Review of the Literature. Journal of the Neurological Sciences, 380, 196-199. https://doi.org/10.1016/j.jns.2017.07.037

The Guardian. (2017). Nigeria's Low Ranking in Global Healthcare Index. Retrieved from https://tinyurl.com/y2tzuo6s

The Medical Futurist. (2019). Reverse Innovation: When Disruptive Health Solutions Go West. The Medical Futurist. Retrieved from https://tinyurl.com/y6nhuu4r

TTRN. (2017). Telehealth Innovation: Current Directions and Future Opportunities. Transatlantic Telehealth Research Network. Retrieved from https://tinyurl.com/y5fjk8nn

WHO. (2019). Country Statistics: Nigeria. World Health Organization web site. Retrieved from https://www.who.int/countries/nga/en/ 
World Bank. (2016). Media (R) Evolutions: Convergence Around Mobile Phones in Sub-Saharan Africa. Retrieved from https://tinyurl.com/y546xnm5

Wyber, R., Vaillancourt, S., Perry, W. et al. (2015). Big Data in Global Health: Improving Health in Low-and Middle-Income Countries. Bull World Health Organ., 95, 203-208. https://doi.org/10.2471/BLT.14.139022

Yeung, S. et al. (2019). A Computer Vision System for Deep Learning-based Detection of Patient Mobilization Activities in the ICU. Digital Medicine. Nature.com, https://doi.org/10.1038/s41746-019-0087-z

Yin, R. K. (2018). Case Study Research and Applications: Design and Methods (6th ed.). Sage Publications.

Yu, D., \& Hang, C. C. (2010). A Reflective Review of Disruptive Innovation Theory. International Journal of Management Reviews, 12(4), 435-452. https://doi.org/10.1111/j.1468-2370.2009.00272.x

\section{Appendix A}

\section{List of participants who have contributed their insights in the online survey.}

In the table, here below, are reported the profiles of the Nigerian professionals (research sample) who have contributed their answers to the online survey in the period March-May 2019. All these participants are highly skilled experts in their own professional field; a number of them have international work and higher education experience, and most of them hold master and doctoral degrees. 


\begin{tabular}{|c|c|c|c|}
\hline Last name & First name & Age & Occupation \\
\hline Ada & Mbang & 43 & Medical doctor \\
\hline Adeniran & Abosede & 35 & HR professional \\
\hline Agege & Osagie & 32 & Operations officer \\
\hline Bakare & Abdul' Azeez & 44 & Agent network manager \\
\hline Bamkole & Peter & 57 & Entrepreneur \\
\hline Bankole-Oki & Abioye & 45 & Entrepreneur \\
\hline Butau & William & 46 & Chartered accountant \\
\hline Carpe & Edward & 42 & Medical doctor \\
\hline Chinedu & Chude Sylvester & 35 & Business development expert \\
\hline Danladi & Simon & 36 & Chief technology officer/civil servant \\
\hline Ebereonwu & Charles & 49 & Communication executive \\
\hline Egoro & Ubong & 35 & Managing director/CEO \\
\hline Ejike & Chukwunonso & 39 & Professor of medical biochemistry \\
\hline Gbenoba & Justice & 45 & Banking manager \\
\hline Jumoke & Dare-Lawrence & 38 & Civil servant \\
\hline Nkanta & Ubong & 40 & Head of customer experience in the health insurance industry \\
\hline Nwachukwu & Chioma & 45 & Registered nurse \\
\hline Odoemene & Kelvin & 39 & Information technology expert \\
\hline Ofili & Onyeka Uche & N.A. & Technology consulting professional \\
\hline Ogbolu & Onuwa & 59 & Entrepreneur \\
\hline Ogundele & Adefemi & 41 & Head of finance business management, Banking industry \\
\hline Ohiowele & Ehizojie & 42 & Group head of transport and logistics in the banking industry \\
\hline Okerezi & Hope & 52 & Hospital administrator and registered nurse at a pediatric hospital \\
\hline Okonta & Chukwuemeke & 51 & Entrepreneur \\
\hline Ojinta & Roseline & 48 & Higher Education Professional \\
\hline Oladimeji & Olawale & 38 & Life and health coach \\
\hline Olayiwole & Gbenga & 40 & Public health consultant \\
\hline Oloke & Tiffany & 44 & Senior specialist in strategic sourcing \\
\hline Olumide & Gloria & 55 & Pharmacist and head of pharmacy unit of a hospital \\
\hline Onajin-Obembe & Bisola & 54 & $\begin{array}{l}\text { Anaesthesiologist and council member, World Federation of } \\
\text { Societies of Anaesthesiologists }\end{array}$ \\
\hline Onigbanjo & Adeyinka & 54 & Head of investment $\&$ portfolio management \\
\hline Opaleye & Oluseyi & 39 & Tax accountant at consulting firm \\
\hline Osagie & Uzoezi & 38 & Financial analyst \\
\hline Oshunniyi & Adeleke & 59 & Medical doctor \\
\hline Otuya & Chinyere & 44 & Registered nurse consultant \\
\hline Oweisana & Akpokabowei & N.A. & $\begin{array}{l}\text { Entrepreneurship professor at Federal College of Education Omoku, } \\
\text { River State }\end{array}$ \\
\hline Saseun & Rowland & 52 & Medical doctor and owner of a private hospital \& maternity clinic \\
\hline Thompson Makinde & Lola & 42 & HR professional \\
\hline Umurhohwo & Deseye & 43 & Entreprenuer \\
\hline Yesufu & Abdul Rahman & 42 & CEO investment firm \\
\hline
\end{tabular}

\section{Copyrights}

Copyright for this article is retained by the author, with first publication rights granted to the journal.

This is an open-access article distributed under the terms and conditions of the Creative Commons Attribution license (http://creativecommons.org/licenses/by/4.0/). 